\title{
Gamification of Apollo Lunar Exploration Missions for Learning Engagement
}

\author{
Resubmitted to Entertainment Computing with Minor Revision
}

\begin{abstract}
The design, development, and evaluation of a serious game that gamifies the Apollo lunar exploration missions are presented. The gamification methodology and underlying implementation are motivated by the goal of game-based learning for youth and young adults and the intention to promote their interest in STEM learning. The game is a multidisciplinary composition that simulates a set of lunar roving activities including planning a traversing route, loading communication and science equipment on the lunar rover, and driving the rover to explore the lunar surface. Accurate 3D terrains and spacecraft models are created and used in the game to maintain the high realism of the environment. Every gameplay step reflects real scientific procedures and behaviors that the astronauts performed in the past on the Moon. A usability study is conducted to gather feedback from a group of participants of different ages and with different game experience. The results of the study show the success of fostering great user engagement in STEM learning.
\end{abstract}

Keywords: gamification, serious games, game-based learning, space science, STEM education

\section{Introduction}

Science is now an integral part of modern life. Scientists receiving public funding are obliged to transmit their research to the public. Poliakoff and Webb [1] discussed many factors that influence the scientists' participations in public engagement activities with science and technology. As science is progressively becoming more complicated and specific, it is not easy to find appropriate ways to improve people's knowledge of science and technology; and it is challenging to enhance people's appreciation for the contributions of scientists and engineers to society. The clear and concise way for scientists to present a scientific contribution is to present the contribution itself, which requires a considerable scientific background for the audience, but that is very often not the case for non-domain members. We see too often that tensions exist at the intersection of people's interest and certain scientific findings. In this paper, we introduce a serious game that gamifies the scientific events of Apollo moon missions executed in the late 1960s and early 1970s. The applied gamification enables a bidirectional communication [2] to engage members of the public, especially for youth and young adults, and promotes their interest in learning in the fields of Science, Technology, Engineering and Mathematics (STEM).

Video games have expanded beyond being simply an entertainment medium, to a medium that many researchers and educators believe can help people engage with, learn, and retain content that they otherwise might struggle with. It is not surprising that video games have been used to supplement both in-classroom and informal education [3, 4, 5]. Serious video games allow people to devour information in interactive and entertaining ways, rather than static or one-sided. Deterding et al. [6, 7] defined the term Gamification which is "the use of game design elements in non-game contexts." Gamified applications present emerging phenomena and make the shift from understanding to engagement to learning promotion. Video games serve as a bridge for the communication between scientists and the public. According to the article by Murphy [8], games can motivate, educate, interact with people, and visualize complex scientific facts; as such, games have strong potential for learning engagement.

Space exploration carried out by astronauts has inspired younger generations to study science and engineering. Since the 1950s, NASA has conducted efforts to several space projects such as the Apollo moon missions, the skylab space station, and the space shuttle. Everyone loves to know about the rockets and space travel. According to a report from the National Center for Science and Engineering Statistics [9], Markovich [10] stated that "the percentage of bachelor'sdegree graduates in science and engineering fields peaked in the late 1960s, around the time of the moon landing, but has declined slowly since". To rekindle the youth interest in STEM and prepare them for the growth of STEM occupations, serious games have been made by science museums and space centers $[11,12]$ which allow people to understand space and aerospace missions through play. While those serious games consider a wide range of audience, they sacrifice a significant amount of realistic interactions and lack the strength to present rich contents of scientific achievements.

In this paper, we present the design, development, and evaluation of an Apollo lunar exploration game, which is a singleplayer serious game that imitates the astronauts' science-related experiment and exploratory activities on the Moon. Different from the lunar rover simulators [13, 14], our game challenges players to accomplish a task under victory or loss conditions. As part of an ongoing NASA-inspired, game-based educational project, we have focused on the gamification of a part of the Apollo 16 mission. The gamification methodology and underlying implementation described in this paper are considered as 
a fundamental platform for the ongoing gamification of Apollo mission series. This game targets primarily on youth and young adults. The game design and development require interdisciplinary knowledge and close collaboration among designers, 3D artists, programmers, mechanical engineers, and educators. Besides the contributions in gamification and interdisciplinary collaboration, we also solve the technical challenges of terrain generation and real-time rendering, where big data from Geographic Information System (GIS) is adopted. The game uses accurate lunar terrain data and detailed 3D spacecraft models to correctly represent historical facts. Besides allowing the traverse of the lunar surface regions discovered in the past, the game also allows players to explore undocumented regions. The evaluation includes an empirical assessment of the game usability and a study of its implication for educational values.

The rest of the paper is organized as follows. Section 2 reviews related work in serious games. Section 3 provides the details of gamification and game design. Section 4 presents solutions to the challenges of lunar terrain generation and realtime rendering. Section 5 describes the challenges of 3D rover modeling. Section 6 presents the evaluation of usability study and analysis of learning engagement. Section 7 concludes our work and proposes future work.

\section{Previous Work in Serious Games}

Serious games have become a popular form to promote learning engagement. Djaouti et al. [15] discussed the origins of serious games in both digital and non-digital forms. Sawyer and Rejeski [16] introduced gaming concepts and technology for non-entertainment purposes. Serious games date back to the 1950s such as computer-supported war games for military training [17]. It was only during recent decades that video games and simulations were widely used in education.

Squire [18] studied in-classroom educational uses of Civilization III which was a popular historical simulation game. He argued that school cultures could be changed to motivate students to investigate questions within a gaming system. Watson et al. [19] presented a case study of using a video game to teach about World War II in a high school history class. With the game the class was changed from a teacher-centered environment to a student-centered learning environment, and the game made students more engaged to the contents. Ricciardi and Paolis [20] provided a comprehensive review of serious games developed for health professions. They concluded that serious gaming technology provided a useful training approach, but serious games were not widespread in healthcare yet as they expected. Luz et al. [21] presented a health-related game that combined the information delivery and a simulation of disease dynamics to support health promotions. Games were also used in other educational areas, such as art history [22], finance [23], relationship [24, 25], culture [26], and training [27, 28].

STEM-related games were incorporated into curricula in educational institutions. NASA Education released an online video game called Moonbase Alpha [29], a future exploration game that simulated the event of colonization after human return to the Moon. The game allowed players to work in a futur- istic 3-D lunar environment. Mayo [30] reviewed the learning outcomes of using video games to teach STEM disciplines. He concluded that game-based learning had the potential to simultaneously educate a large group of students, but games were not largely adopted at the time because of the infrastructural obstacles like the lack of student quantity, low quality of academic games, and disruptive business models. Miller et al. [31] studied the students' knowledge gained in science from playing an online forensic science game. They concluded that serious games in school environments were good for classroom discussion and hands-on activities. Miller [32] reviewed several online simulations and role-play games and proposed a few ways how games may encourage students to pursue STEM careers.

The success of serious games relies on appropriate gamification. Yusoff et al. [33] presented a conceptual model as guidelines of serious game development to help measure achievements in regards to the satisfaction of learning expectations. Deterding et al. $[6,7]$ defined the term gamification and investigated the origins of the term in industry and HCI research. They concluded that gamified applications furnished insight into novel. Li et al. [34] presented GamiCAD which was a game interactively teaching first-time users how to use AutoCAD software. The extensive real-time visual and audio feedback in the game were the significant contributions of gamification. Kim [35] provided a systematic study of the gamification methods for library services and programs. The work considered a variety of variables rather than just treating the games as an instructional approach. Dergousoff and Mandryk [36] presented a smartphone-based game for crowdsourcing, where players were motivated by in-game rewards to do the experimental tasks.

\section{Lunar Exploration Tasks and Gamification}

Our game combines information about the Moon, scientific principles, realism, and intelligent applications of technology to offer an engaging interaction and play. It challenges users to accomplish a lunar exploration mission, which imitates what astronauts did with the Apollo Lunar Roving Vehicle (LRV). The gameplay mechanics is developed based on the actual activities performed by the astronauts on the lunar surface. The gamification focuses on the imitations of the activities listed in Table 1. We develop three gameplay phases including Traverse Planing, Equipment Loading, and Rover Driving. The player's performance is rated at the end of the game and displayed on a scoreboard. An overview of the game development is illustrated in Figure 1. Subsection 3.1-3.3 provide the details of the design of the gameplay phases, each including real scenarios of the Apollo mission and the gamification methods. Subsection 3.4 describes the scoring metric.

\subsection{Traverse Planning}

The actual traversing routines were designed by the Mission Control in Houston as general guides for the astronauts to follow. The coordinates of landing sites vary in different Apollo missions. For the Apollo 16 mission, the landing site was close 


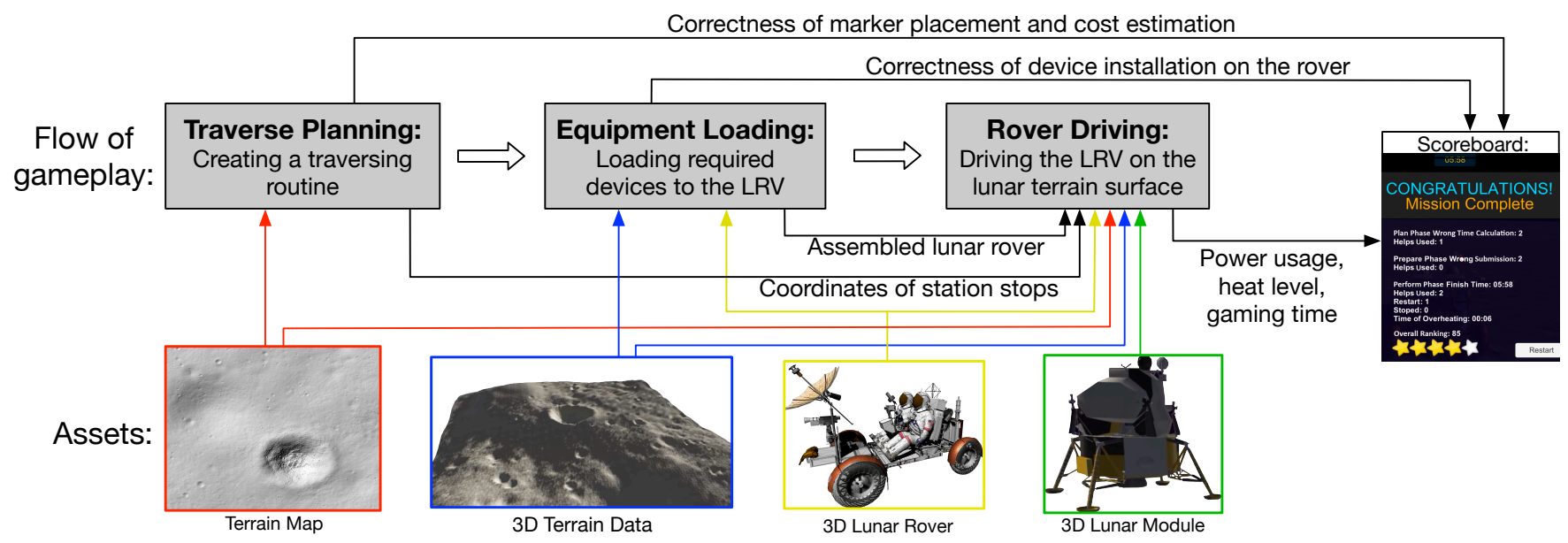

Figure 1: Overview of the game development.

Table 1: Typical activities that astronauts performed in an EVA Typical activities in an EVA

- Communicating with the Mission Control.

- Estimating the traversing distance and travel time.

- Learning about devices/parts and loading required ones to the LRV.

- Driving the LRV with a special steering controller.

- Monitoring and controlling overheating issues.

- Using the navigation system to find station stops while driving.

- Managing the driving time.

- Viewing geomorphic features of the lunar surface.

to the Descartes Crater, which is a region important for geologists to study lunar volcanism and its contribution to the lunar highland evolution [37]. Astronauts remained on the lunar surface for 73 hours, including three 7-hour Extra Vehicular Activities (EVAs). A traversing path was made in each EVA and composed of several station stops. The astronauts drove to each station stop to collect soil samples or deploy scientific experiments.

The Traverse Planning phase mimics the processes of listening to the Mission Control, creating a traversing path, and estimating the travel time. The user receives the coordinates of station stops from a simulated Mission Control, and creates the route by placing markers on a lunar map. The markers represent the locations of station stops. A 7-hour EVA traversing path would be too long for gaming and would not give a pleasant rover driving experience. To avoid being boring or making the user feel tired of driving, our game uses a short path which takes a few minutes of driving to finish - that allows the user to have just enough attention to grasp the key points.

Operational instructions and the coordinates of station stops are displayed in the form of screen-rolling texts. Figure 2(a) is a screenshot of the Traverse Planning phase. The dialogue panel in the bottom-left corner of the interface lists the information of totally 4 station stops. The coordinates are latitude and longitude values. The lunar map is overlaid with a $10 \times 10$ reference grid, where line labels are coded in latitude and longitude to help the user identify marker locations.
The interface can get users quickly into the interactions with the map and the geographic coordinate system. The interface does not have many UI elements, because overlaying many UI elements or placing them on a $2 \mathrm{D}$ hub plane would take the user's attention away from the content. A new marker is created by mouse-clicking a location on the map. Markers are draggable and clamped to map boundaries. If the marker is not at the correct location, the user can re-click on the map to relocate it. When the marker is moved to the correct location, the color of the marker will change indicating it is no longer movable. The dialogue panel provides real-time text feedback to help the user correct any mistakes made in the process of marker placement.

When all markers are correctly placed, the user is promoted to calculate the travel time, using the distance of the traversing route (kilometers) and the given maximum driving speed $(\mathrm{km} / \mathrm{hr})$. A calculator appears on the interface for the user to use. The user has two chances to enter answers. The game will continue to the next phase when either the correct time is entered or the user has entered wrong answers twice.

\subsection{Equipment Loading}

The astronauts had to load required communication and science devices on the LRV for the scientific activities they would perform at the station stops. Consultations with the scientists in the Mission Control over the radio were usually needed in order to decide what particular devices should be installed on the LRV. The LRV had to be deployed from the Lunar Module before driving. According to Young's book [38], the LRV was a self-propelled, manually controlled four-wheel vehicle for the use of transporting astronauts and equipment on the lunar surface. Some devices were not movable, such as Display and Control Console (DCC) (see Figure 3), while others such as $\mathrm{TV}$, antennas, rake, and potentiometer were attachable and removable.

In the game, the interface of the Equipment Loading phase illustrates mechanical features of the LRV, displays 3D shapes of the devices, and explains their usages. In this phase, the 


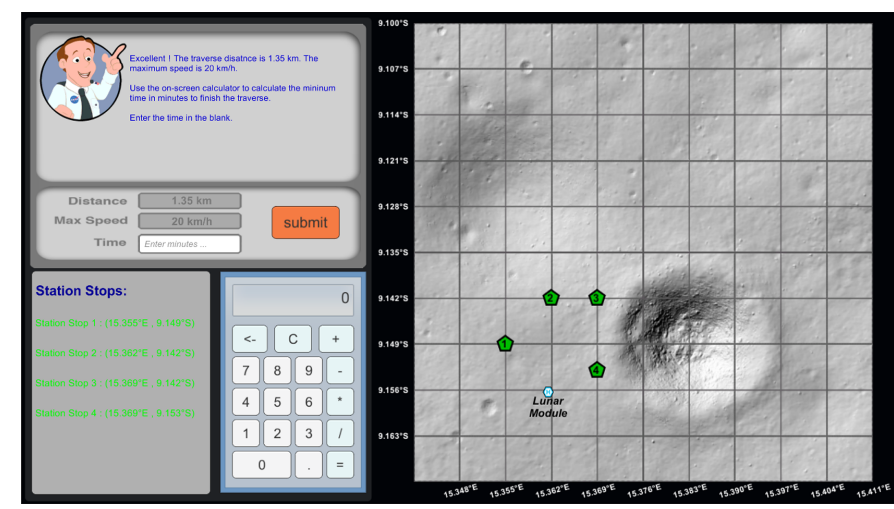

(a)

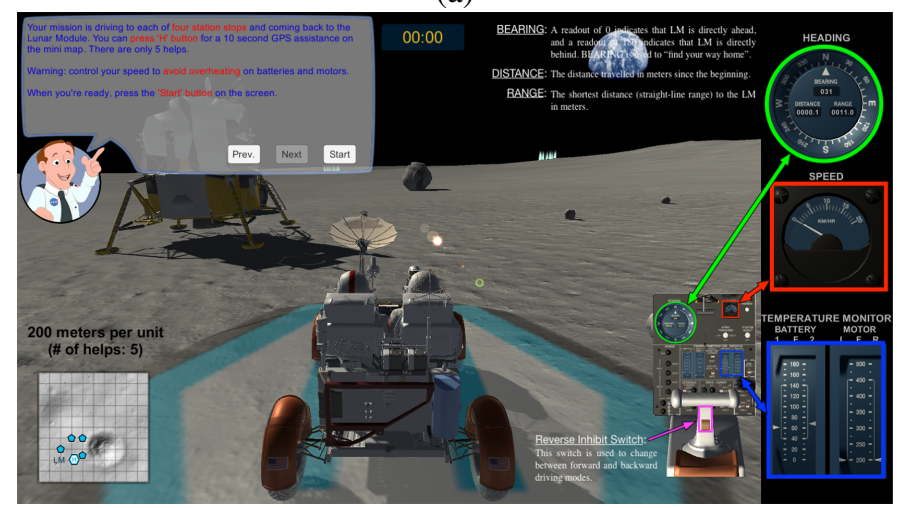

(c)

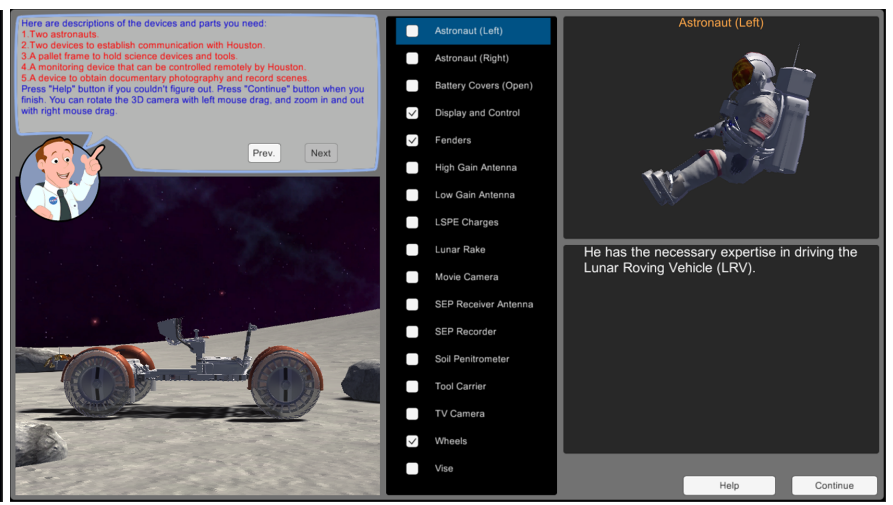

(b)

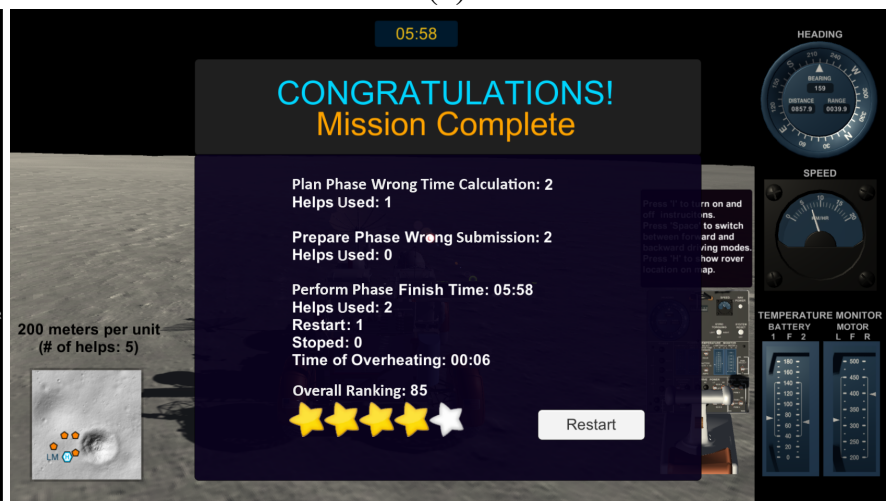

(d)

Figure 2: Screenshots of three gameplay phases and the view of the score board. (a) is a screenshot of the plan phase, where four markers have been placed correctly and the user will need to enter the traverse time with the calculator. (b) is a screen shot of the prepare phase that shows a list of rover parts or devices. (c) is a screen shot of the perform phase. The on-screen instruction is activated to explain the usage. (d) is the scoreboard view that shows the user's performance details and gives an overall ranking.

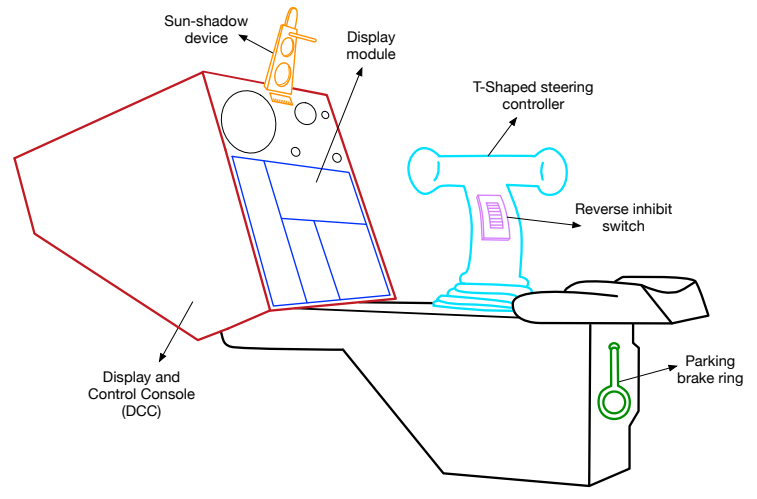

Figure 3: Illustration of the steering controller and the DCC.

immovable parts and devices have been loaded on the LRV, including the frame, wheels, fenders, seats, seat belts, and DCC. The user will need to identify a set of devices based on the requirements from the simulated Mission Control. Figure 2(b) is a screenshot of the Equipment Loading phase. The full list of devices is shown in an inventory, where the checkboxes of those immovable devices have been checked and other attachable devices are not. A learning process is embedded through interactions with the inventory. When a device is selected, its 3D shape and usage are shown in a separate panel, from which the user will learn about the functionality. To help the user observe the 3D shapes, The 3D view can be rotated to any angle to observe the 3D shape.

The assembled LRV model is rendered in a separate window. Checking a device in the inventory will make the device appear on the LRV so that the user can know where the device is installed exactly and its relationship with other components. The viewing camera has a 360 auto-rotate feature with the view pivot at the center of the LRV. It can also be zoomed in or out for close-up or long-distance views. This process provides both entertaining and educational values.

While users are expected to figure out themselves what the required devices are, there is a help button on the interface for users to acquire hints. To fairly measure the performance of users' play, the overall score reported at the end will be deducted if the help button is used (see Section 3.4).

\subsection{Rover Driving}

The Rover Driving phase offers driving experience on the lunar surface. The simulation of the rover's suspension system is implemented. Besides responding to $1 / 6$ Earth gravity and dodging craters, the experience includes (1) performing steering and braking operations, (2) using the navigation module to find station stops, (3) using a mini map to determine the driving 
direction, and (4) monitoring and controlling battery and motor temperatures.

Steering and braking. LRV Operations Handbook [39] described a T-shaped controller situated between the two seats and in company with the DCC. Figure 3 illustrates the controller and the DCC. The controller has a reverse inhibit switch to prevent from inadvertently placing the vehicle in reverse. When the switch is in the down position, pushing the controller accelerates the LRV in the forward direction, and pulling it backward reduces the speed until the vehicle stops. When the switch is in the up position, the vehicle can only move in the reversed direction; pulling the controller increases the backward speed, and pushing it reduces the speed until the vehicle stops. Moving the controller left and right increase the left or right steering angle to turn the vehicle. Pulling the parking ring activates the parking brake. In the game, we map these driving operations on a flight stick. Pressing a specific button on the flight stick mimics the activation of the reverse inhibit switch.

Navigation module. In the DCC, a computer behind the navigation module was designed to track the distance traveled since the vehicle left the Lunar Module. A sun-shadow device used the sun to give a heading direction by rotating the heading dial. The direction and distance information were displayed in the navigation module (see Figure 3). The navigation module also included a speed meter displaying the driving speed and temperature meters showing the heat levels of batteries and motors.

The gaming scene of the Rover Driving phase is in a 3D environment. The main camera follows the movement of the LRV. Fitting UI elements on the 3D object of the DCC would result in poor readability. Texts on the UI elements would be too small to read through the camera. In this game, we place the UI elements of the heading dial, the speed meter, and temperature meters in a $2 \mathrm{D}$ hub plane to the right of the main window (see Figure 2(c)).

Mini map and GPS tracking. The main window contains a mini map which displays the location of the Lunar Module, the locations of station stops, and geological features. In the era of the Apollo missions, the LRV did not have a GPS-like system. In this game, users need to use the navigation system to find the driving direction. Since there is no landmarks or cues on the lunar surface, users may misjudge the driving direction. We implement a LRV tracking system as a special help for the users to get back to the traverse routine when they lose the direction. A specific button on the flight stick is used to activate the tracking system, so the location of the LRV and the driving direction will show on the mini map for 10 seconds; however, this feature can only be used for a maximum of 5 times. Using the GPS tracking feature is not encouraged since we want users to learn the usage of the navigation system of the LRV. The more times the users use the tracking system, the lower their scores will be at the end of the game.

Battery and motor temperatures. The LRV was designed to have two batteries, which provided power for motors and communication instruments. During the driving, batteries and motors produced heat. The speed and acceleration of the LRV had to be appropriately controlled in order to avoid overheating issues; otherwise the LRV could be forced to stop. In the game, users need to monitor temperature changes. The temperature meters are organized in the same 2D hub plane as other UI elements. In the actual lunar roving activities, heat from the sun and reradiated heat from the Moon may expedite overheating during the traverse of a 7-hour EVA. In our design we do not consider the affects of such heats because the traverse created in the game only takes a few minutes to finish.

In the game, a warning message requiring to reduce the speed will appear on the screen when a battery or motor overheats. If the heat level continuous increasing, the LRV will be forced to stop until the battery and motor cools down to a normal level. This design intends to motivate users to be aware of the heat from the continuous consumption of battery power and the generation of motor torque. Simulators of a real thermal model [40, 41] might lead to high computational costs, which prevent users from real-time gaming experience. In this game, we implement a simple and effective solution, as described below, to mimic thermal actions on the batteries and motors. It executes fast and gives users overheating alerts.

$$
\begin{gathered}
T_{B}=T_{B}+\alpha \times H_{B} \\
H_{B}= \begin{cases}\delta H_{B}, & (\text { acc }>0, \text { speed }<15) \\
\delta H_{B} \times\left(1+\left(\frac{\text { speed }}{\text { maxs peed }}\right)^{\beta}\right), & (\text { acc }>0, \text { speed } \geq 15) \\
-h_{B}, & \left(\text { acc }=0, T_{B}>T_{D}\right) \\
0, & \left(\text { acc }=0, T_{B}=T_{D}\right)\end{cases}
\end{gathered}
$$

$$
\delta H_{B}=|a c c| \times h F a c \times\left(1-e \text { Rate }^{n}\right)
$$

Equations 1-3 are executed in the main update loop of the game to compute the battery temperature $\left(T_{B}\right)$. In Equation $1, \alpha$ is a global parameter to determine the percentage contribution of the battery heat $H_{B}$ to $T_{B} . H_{B}$ is calculated based on the acceleration and the speed of the LRV. In Equation 2, acc is the acceleration scalar returned from the roving simulation. $\delta H_{B}$ is the heat generated based on the acc when $a c c>0$. While the LRV accelerates, the battery temperature will increase faster as the speed is greater than $15 \mathrm{KM} / \mathrm{HR}$, by a heat scaling factor controlled by an exponential parameter $\beta$.

The battery has a default temperature $\left(T_{D}\right)$ as the rover engine starts. When $a c c=0$, the battery cools down by a constant amount of heat $\left(h_{B}\right)$, until $T_{B}$ is reduced to $T_{D}$. In Equation 3, eRate is the natural heat escaping rate. $n$ is a thermal controlling parameter. $h F a c$ is the heat converting parameter which is used to linearly map the value to a celsius temperature. Table 2 lists the values assigned to those parameters for our implementation.

Similarly, Equations 4-6 are used to compute the motor temperature. The motor temperature $\left(T_{M}\right)$ is affected by the acceleration of the rover and the slope of the lunar surface. A high sloped surface causes more heat on the motors than a flat surface does. In Equation 6, the value of $h F a c$ varies depending on whether or not the slope exceeds the threshold of dangerSlope. The slope is a scalar which is an Euler angle determined by the surface condition for the LRV to climb up or slide down. If slope < dangerSlope, the base heat factor $k$ is assigned to 
Table 2: The values assigned to the parameters for heat calculation (refer to Equations 1-6)

\begin{tabular}{|c|c|c|c|}
\hline Heat Types & Equations & Parameters & Values \\
\hline \multirow{10}{*}{ Battery } & $\begin{array}{c}\text { Equation 1 } \\
\left(T_{B}\right)\end{array}$ & $\alpha$ & 0.2 \\
\hline & \multirow{5}{*}{$\begin{array}{c}\text { Equation } 2 \\
\left(H_{B}\right)\end{array}$} & speed & $\begin{array}{l}\text { a value in }[0, \text { maxS peed }] \text { returned } \\
\text { from the rover simulation }\end{array}$ \\
\hline & & maxS peed & $20 \mathrm{KM} / \mathrm{HR}$ \\
\hline & & $\beta$ & 2.5 \\
\hline & & $h$ & $0.1^{\circ} \mathrm{C}$ \\
\hline & & $T_{D}$ & a random value in $\left[40^{\circ} \mathrm{C}, 80^{\circ} \mathrm{C}\right]$ \\
\hline & \multirow{4}{*}{$\begin{array}{c}\text { Equation } 3 \\
(\delta H)\end{array}$} & $a c c$ & $\begin{array}{l}\text { a value in }[0,1] \text { returned from } \\
\text { the rover simulation }\end{array}$ \\
\hline & & hFac & $30^{\circ} \mathrm{C}$ \\
\hline & & eRate & $98 \%$ \\
\hline & & $n$ & $\begin{array}{l}\text { initialized with } 1 \text {; } \\
\text { increased by } 1 \text { every } 10 \text { seconds; } \\
\text { reset to } 1 \text { when speed }=0 \text {. }\end{array}$ \\
\hline \multirow{10}{*}{ Motor } & $\begin{array}{c}\text { Equation } 4 \\
\left(T_{M}\right)\end{array}$ & $\alpha$ & 0.2 \\
\hline & \multirow{5}{*}{$\begin{array}{c}\text { Equation } 5 \\
\left(H_{M}\right)\end{array}$} & $a c c$ & $\begin{array}{l}\text { a value in }[0,1] \text { returned from } \\
\text { the rover simulation }\end{array}$ \\
\hline & & eRate & $95 \%$ \\
\hline & & $n$ & $\begin{array}{l}\text { initialized with } 1 \text {; } \\
\text { increased by } 1 \text { every } 10 \text { seconds; } \\
\text { reset to } 1 \text { when speed }=0 \text {. }\end{array}$ \\
\hline & & $h$ & $0.2^{\circ} \mathrm{C}$ \\
\hline & & $T_{D}$ & $0^{\circ} \mathrm{C}$ \\
\hline & \multirow{4}{*}{$\begin{array}{c}\text { Equation } 6 \\
(h F a c)\end{array}$} & slope & $\begin{array}{l}\text { a value in }[0, \text { dangerS lope }] \text { returned } \\
\text { from the physical simulation }\end{array}$ \\
\hline & & dangerSlope & $30^{\circ}$ \\
\hline & & $k$ & 20 \\
\hline & & $\beta$ & 65 \\
\hline
\end{tabular}

$h F a c$; otherwise it is increased by a certain amount with respect to the slope ratio. $\beta$ is a scaling parameter to adjust the percentage contribution of the slope ratio to $h \mathrm{Fac}$.

$$
\begin{gathered}
T_{M}=T_{M}+\alpha \times H_{M} \\
H_{M}= \begin{cases}a c c \times h F a c \times\left(1-e \text { Rate }^{n}\right), & (\text { acc }>0) \\
-h, & \left(a c c=0, T_{M}>T_{D}\right) \\
0, & \left(a c c=0, T_{M}=T_{D}\right)\end{cases} \\
h F a c= \begin{cases}k, & (\text { slope }<\text { dangerSlope }) \\
k+\beta \times \frac{\text { slope }}{\text { dangerSlope }}, & (\text { slope } \geq \text { dangerSlope })\end{cases}
\end{gathered}
$$

\subsection{Scoring Metric}

A scoreboard displays the user's performance upon game completion. Based on the score calculated in Equation 7, it gives an overall rank that has responses ranging from 0 to 5 stars (see Figure 2(d)). Table 3 shows a list of parameters used

\begin{tabular}{|c|c|}
\hline Phases & Scoring Parameters \\
\hline $\begin{array}{l}\text { Traverse } \\
\text { Planning }\end{array}$ & $\begin{array}{l}n_{\text {wrong } M} \text { - the number of mistakes made during maker placement. } \\
n_{\text {help }} \text { - the number of helps used to fix the mistakes. } \\
n_{\text {wrong } T} \text { - the number of times of wrong traversing time entered. }\end{array}$ \\
\hline $\begin{array}{l}\text { Equipment } \\
\text { Loading }\end{array}$ & $\begin{array}{l}n_{\text {wrongR }} \text { - the number of submissions of wrongly assembled LRV. } \\
n_{h_{\text {help }}}-\text { the number of helps used to identify parts/devices. }\end{array}$ \\
\hline $\begin{array}{l}\text { Rover } \\
\text { Driving }\end{array}$ & $\begin{array}{l}t_{\text {traverse }}-\text { traverse time in seconds. } \\
t_{\text {heat }}-\text { overheating time in seconds. } \\
n_{\text {stop }}-\text { the number of times of being forced to stop. } \\
n_{\text {gps }}-\text { the number of uses of the rover tracking system. } \\
n_{\text {restart }} \text { - the number of times of the phase restarted. }\end{array}$ \\
\hline
\end{tabular}
for score calculation.
Table 3: A list of parameters used for score calculation.

$$
\begin{array}{r}
\text { Score }=\operatorname{Max}\left(0,5-n_{\text {wrong } M}-n_{\text {help }_{1}}\right)+\operatorname{Max}\left(0,10-n_{\text {wrongT }}\right)+ \\
\operatorname{Max}\left(0,10-2 \times n_{\text {wrongR }}\right)+\operatorname{Max}\left(0,10-n_{\text {help }_{2}}\right)+ \\
\operatorname{Max}\left(0,10-2 \times n_{\text {gps }}\right)+\operatorname{Max}\left(0,\left(9-3 \times n_{\text {stop }}\right)+\right. \\
\operatorname{Max}\left(0, \operatorname{Min}\left(25,25-2 \times\left(t_{\text {traverse }} / 60-4\right)\right)+\right. \\
\operatorname{Max}\left(0, \operatorname{Min}\left(16,16-2 \times t_{\text {heat }} / 60\right)+\operatorname{Max}\left(0,5-n_{\text {restart }}\right)\right.
\end{array}
$$

\section{3D Lunar Terrain Generation}

Visualization of the Earth, Moon, or solar system has been used in many space themed games. For example, NASA's Eyes [42] supported an interactive exploration of the Earth and solar system using visualization technologies, but it had to adopt basic imagery maps for planets in order to render at high frame rates. In our game, high quality $3 \mathrm{D}$ terrain models are needed to represent rich geological information of the lunar surface, as well as for the requirements of accurate collision simulation and ground slope detection. For those purposes, we use Lunar Reconnaissance Orbiter (LRO) datasets [43] to generate the terrain model. This section describes our method of terrain generation which produces a 3D model meeting the demands on both visual fidelity and rendering performance.

\subsection{Global and Regional DEM}

The LRO instruments have been in operation since 2009 for explorative missions and have captured data such as temperature maps, geodetic grid, and high-resolution images. These data are necessary for future human and robotic lunar missions. The Lunar Mapping and Modeling Program (LMMP) at NASA [44] provides topographic maps of the Moon which were generated based on the LRO datasets. The topographic maps are saved as a Digital Elevation Model (DEM) which represents height information in equirectangular projection. The DEM provided by LMMP is a global map covering the entire surface of the Moon, but the resolution is not high enough to show the topographical detail in close-up views (see Figure 4). The configuration of this global DEM is 256 pixels per degree and at a scale of 118 meters per pixel with interpolation at missing data points. We use this global DEM in the game to construct far-away landscapes.

Higher resolution data is available in a regional DEM archived at Arizona State University [45]. A regional DEM was 


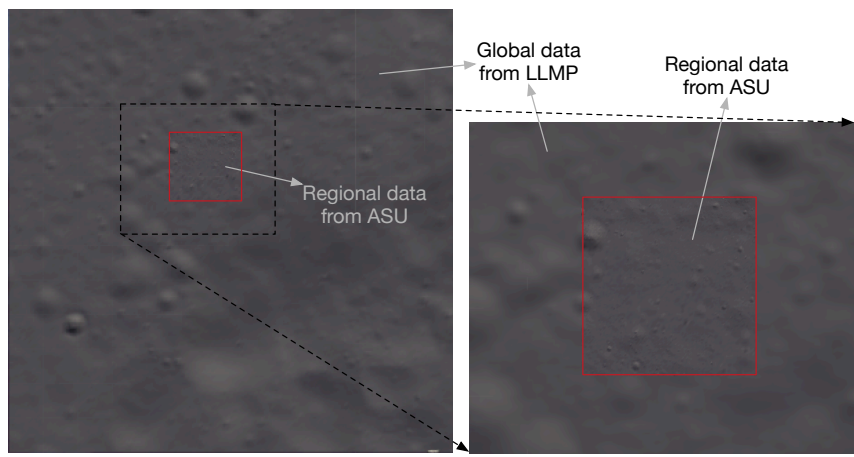

Figure 4: The use of global and regional DEMs. The image on the right is the zoom-in view of the dotted square area. The global DEM is from LLMP at NASA where one pixel represents 118 meters. The regional DEM from ASU used in the red square is the terrain data used for rover traversal. One pixel in the regional DEM represents 2 meters. The global DEM is much blurrier than the regional DEM and used in the game to enhance the visual fidelity of far-away landscapes.

created using the image obtained from Narrow Angle Cameras (NACs) on the LRO instruments. The regional DEM used in our game provides the topography at a scale of 2 meters per pixel (see the red squared region in Figure 4).

\section{2. $3 D$ Construction Using Heightmap}

In order to generate the 3D terrain model, the DEM is first converted to a heightmap where height information is represented as normalized grayscale values. Lighter values indicate higher elevations. Comparing to a polygonal mesh, a heightmap requires much less memory on storage and supports faster rendering. Most of modern game engines have a build-in terrain system that uses the heightmap to create a precise terrain surface. Figure 5 shows the workflow for generating a 3D terrain model.

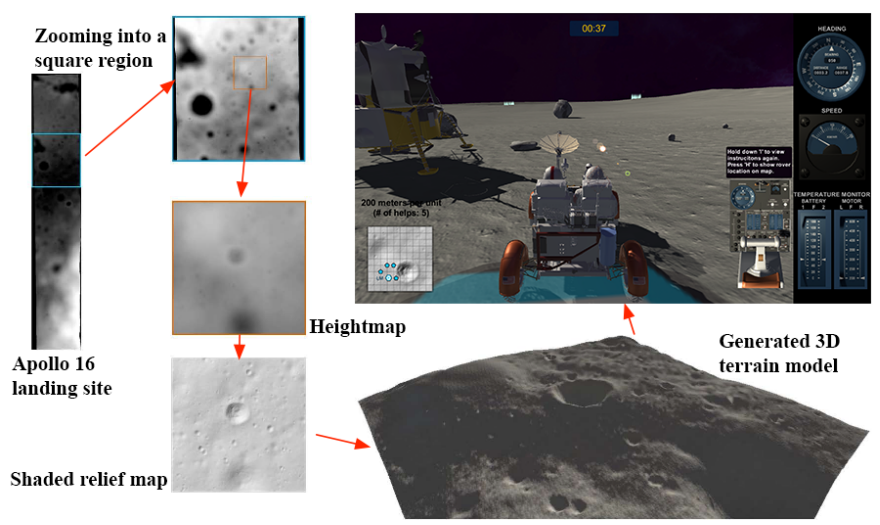

Figure 5: The workflow of 3D terrain generation. The DEM data is converted to a heightmap, and then the shaded relief map is produced in order to generate the $3 \mathrm{D}$ terrain. As shown in the shaded relief map and in the 3D model, the areas with steep slopes are rough and contain significant geological features.

We adopted a GIS tool called QGIS [46] to crop the DEM and export a heightmap. QGIS allows us to use latitude and longitude values to specify and view the cropped region. It also has a layer panel which displays the minimum and maximum elevations (in meters) from the referenced spherical surface. The size of the heightmap is set based on the requirement of Unity game engine, which requires a heightmap dimension has to be a power of 2 plus 1 , such as $513 \times 513$ and $1025 \times 1025$. The "plus 1 " ensures the pixel values of the heightmap line up to the vertices that are dynamically created for tessellation on the terrain surface.

A DEM may contain noise which is a random occurrence of irrelevant values. Noise can perturb measures of the surface shape such as slopes and mountains, and disrupt the natural appearance of the terrain. Before using the DEM to generate the 3D terrain, we adopt the denoising method presented by Sun et al. [47] to remove the noise in it, while preserving features such as sharp edges and corners. The desired degree of denoising is achieved by iteratively smoothing face normals in heightmap space.

\section{LRV Model}

The game uses the 3D LRV model same as the one in the NASA Virtual LRV project [48]. It contains loosely connected, asymmetrically tessellated, intertwining detailed objects with widely varying spatial ratio and complex topologies (see Figure 6). The model consists of over 900 thousand triangles and over 400 thousand vertices. The LRV model is too complex and cannot be used to perform the thermal and roving simulation. We adopted the idea of Progressive Meshes [49, 50, 51] to reduce the geometric complexity while maintaining high visual fidelity for the appearance. From the viewpoint of camera, the objects far away from the camera are simplified so they have less geometric detail than those close to the camera. We also remove invisible objects such as the surface radiators under the dust covers.

The modules on the DCC including the heading dial, speed meter and temperature meters are not represented with enough geometric detail in the LRV model. We create high detailed 3D models for those modules so that they can be used in the 2D hub plane in the Rover Driving phase. As shown in Figure 6, each model is created for accurate mechanical movements and wrapped with precise textures.

The game scene of the Rover Driving phase is rendered realistically with appropriate shadows and shades on the terrain and LRV models. We also add visual effects to simulate the environment of the Moon, including sun glare and dark out space, thin dust layers on the moon's surface, etc. A realistic rendering result is an important characteristic of our game that drives user actions in an immersive environment.

\section{Usability Study}

Our usability study is designed to find out how well the game serves the educational purpose and effectiveness of delivering scientific facts to people. We want to know whether or not users are motivated after playing the game to learn more about the science facts presented in the game. Furthermore, we want to know whether or not their interest is increased in STEM learning. This section describes the design of usability study and assesses the results. 


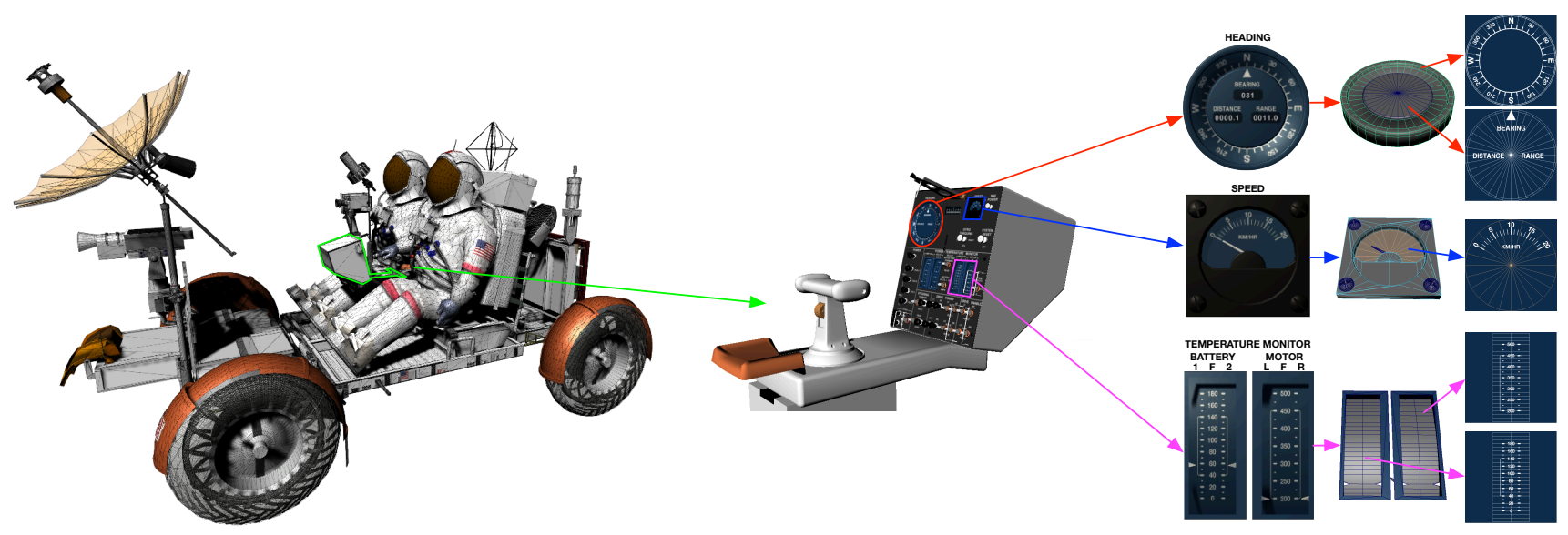

Figure 6: The 3D LRV model with shaded wireframes that contains more than 900 thousand polygons.

\subsection{Expert Evaluation}

As regards the realism of the game, we received positive feedback from the experts who worked on the Apollo missions in 1970s. The physical simulation of the rover reacting to the lunar terrain is consistent to the facts of Apollo lunar roving missions. We also obtained comments from commercial game studios about the design of gameplay mechanics which helped us improve the game quality. We were informed that a VR conversion of this game in the near future would likely be loved by players.

\subsection{Participants and Methodology}

Participation of this usability study was completely voluntary. We recruited 30 participants including both males and females between the ages of 19 and 38. They all had driving experience and received necessary math and general science training. They signed up for the usability study either through an online reservation link or by sending an email to the investigators of this study. The study was performed in a research lab room in the university. During the study the room was occupied only by the participant and one of the investigators. A consent form was presented to the participant prior to the study. It included the goal, an overview of the game, procedures, and consent information regarding confidentiality, discomforts and freedom to withdraw.

A demographic questionnaire was filled by the participant. It was used to learn about the participant's previous attitudes, feelings and experience in playing video games. The participant then played the game on a desktop computer with a mouse, a keyboard and a flight stick. We did not give a tutorial session since the game has embedded instructions that guide the participant through the game step-by-step and explain the gameplay elements. During the play, the investigator was able to answer the questions regarding the controls of the game. After finishing the game, the participant filled a qualitative questionnaire. Questions about anxiety, depression and how much the participant worried or ruminated about his or her feelings were also asked in the questionnaire. The entire session of the study for one participant took 40-50 minutes. Figure 7 are the pictures taken during the study.

\subsection{Questionnaires}

The demographic questionnaire is composed of questions in 5 categories which are listed below:

- Do you currently play video games? How long have been playing them? How often (approximately) do you play them? And would you be interested in playing video games in the future?

- How did you get started playing video games? who or what did motivate you to play?

- Do you prefer to play with or without people around or through internet without people physically around you?

- What the gaming platforms/consoles on which you play games?

- What are the top 3 video game categories that you enjoy to play? and what are the top 5 video games that you enjoy to play?

The qualitative questionnaire has a total of 10 questions. 9 of them request participants to give a rating on a 10-point Likert scale. Each question also has space for comments. The goal is to evaluate (1) if the game efficiently embeds many science-related instructions, (2) if the game effectively represents the knowledge of historical lunar exploration missions, and (3) if the game motivates participants to learn more about Apollo missions or related topics after playing it. Table 4 lists the questions and shows the ratings. Q1 and Q2 are to evaluate the game as a whole; Q3-Q6 ask for opinions in learning the subject; Q7-Q9 are the questions about individual play phases; Q10 is to find out if participants feel depressed or helpless as the similar feelings the astronauts might have since they were the only life form on the Moon at the time.

\subsection{Results}

\subsubsection{Results of the demographic questionnaire}

All participants had the experience of playing video games. Most of them preferred to play games with friends or online. 

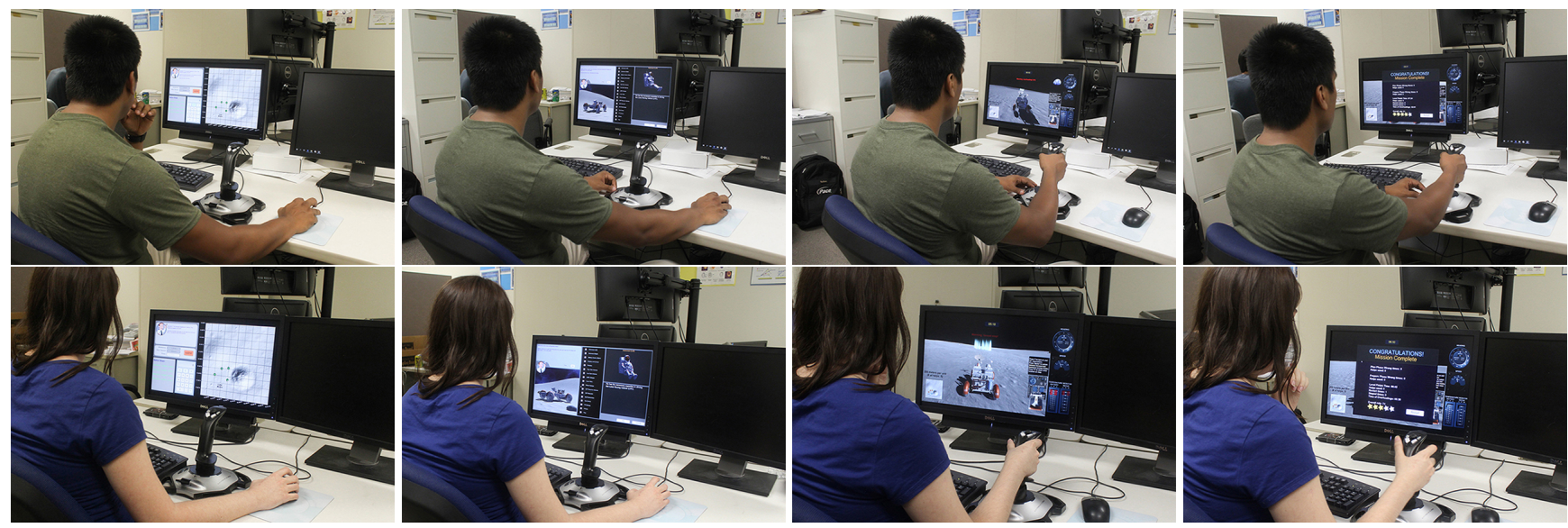

Figure 7: Pictures of the usability study showing the participants played through the game.

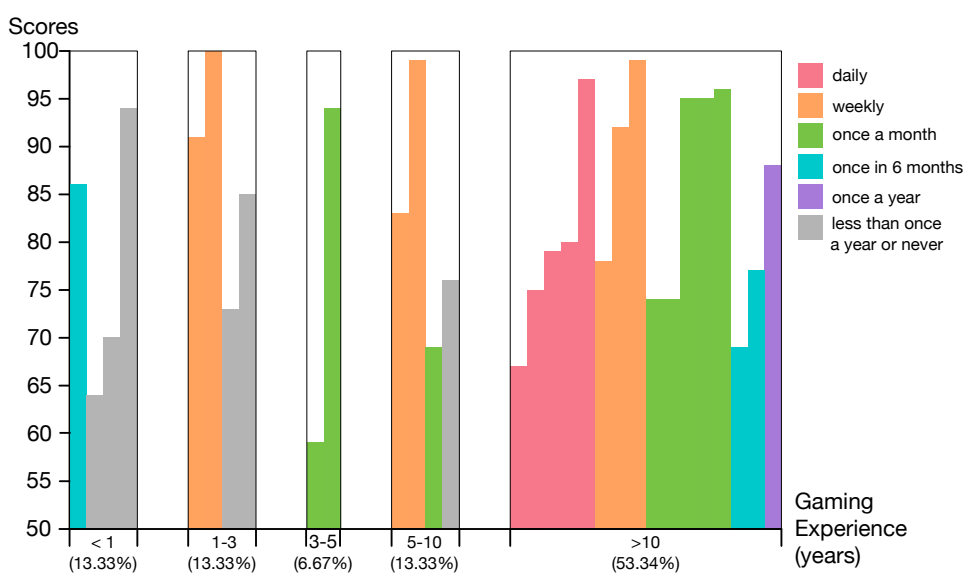

(a)

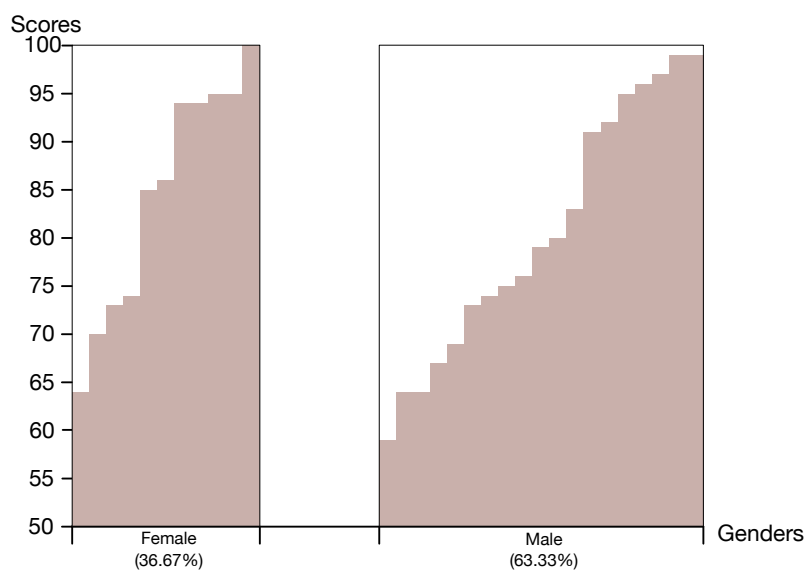

(b)

Figure 8: (a) The scores with respect to gaming experience; (b) The scores with respect to genders.

Figure 8 illustrates the scores that they obtained in this game with respect to their gaming experience and genders. The score metric has responses ranging from 0 to 100 . More than half of the participants $(53.34 \%)$ have been playing video games for more than 10 years. From the results, we did not find a significant association between the performance of the participants in this game (scores) and their gaming experience. As indicated in Figure 8, there were two participants who play video games daily for more than 10 years. One participant scored 97 in this game and the other one scored 79 . We found that the participants who enjoyed strategy or racing games scored higher in this game than those who enjoyed puzzle, action or first-person shooting games. The average score of male participants was 80.63 , and the average score of female participants was 84.55. We performed an unequal variance t-test [52] between the female and male scores. The t-test result is not statistically significant. The $\mathrm{P}$ value in the t-test result equals 0.423 which indicates that the game is likely good for both female and male users, rather than appropriate for one group over the other.

\subsubsection{Gameplay time}

The average time to finish the game was $7^{\prime} 52^{\prime \prime}$. Female participants finished the game faster than male participants. The average time of female participants was $7^{\prime} 04^{\prime \prime}$; the average time of male participants was $8^{\prime} 22^{\prime \prime}$. Note that finishing the game faster does not guarantee a higher score. For example, one participant finished the game at $77^{\prime} 55^{\prime \prime}$ with a score of 74 and the other one finished the game at $9^{\prime} 50^{\prime \prime}$ with a score of 86 . The former participant finished faster but used 2 helps and the overheating forced the LRV to stop for $1^{\prime} 55^{\prime \prime}$. The later participant took longer time to finish but no help was used and the LRV was forced to stop for only $20^{\prime \prime}$. One participant commented on the score metric that "You need to control the car correctly. Driving fast is not always good."

\subsubsection{Results of the qualitative questionnaire}

Overall evaluation. As regards Q1 and Q2, positive comments highlight that the way of play provides a smooth learning curve and the guidelines are appropriate. Some participants expressed that they were impressed with the graphics, the quality of the $3 \mathrm{D}$ models and the level of realism created in the game. 
Table 4: The results of the qualitative questionnaire.

\begin{tabular}{|c|c|c|c|c|c|c|c|}
\hline \multirow[b]{2}{*}{ \# } & \multirow[b]{2}{*}{ Questions } & \multirow[b]{2}{*}{ Liket Scale Points } & \multicolumn{5}{|c|}{ Results } \\
\hline & & & Max. & Min. & Avg. & Median & $\begin{array}{c}\text { Standard } \\
\text { Deviation }\end{array}$ \\
\hline Q1 & What is your overall impression of the game? & (very negative) $1 \ldots 10$ (very positive) & 10 & 1 & 7.70 & 8.00 & 1.78 \\
\hline Q3 & $\begin{array}{l}\text { After playing the game, did you want to learn more about the } \\
\text { events of lunar roving? }\end{array}$ & (Not at all) $1 \ldots 10$ (very eager to learn more) & 10 & 1 & 6.67 & 7.50 & 3.28 \\
\hline Q4 & $\begin{array}{l}\text { After playing the game, did you think you gained interest in } \\
\text { space science? }\end{array}$ & (No interest gained) $1 \ldots 10$ (lots of interest gained) & 10 & 1 & 6.03 & 7.00 & 2.77 \\
\hline Q5 & $\begin{array}{l}\text { The interfaces, game objects and visual effects presented in the } \\
\text { game made it easy to learn. }\end{array}$ & (Strongly disagree) $1 \ldots 10$ (Strongly agree) & 10 & 3 & 7.47 & 8.00 & 2.24 \\
\hline Q6 & $\begin{array}{l}\text { By playing this game, you gained a full understanding about } \\
\text { how to use the T-shaped hand controller to drive the lunar roving } \\
\text { vehicle. }\end{array}$ & (Strongly disagree) $1 \ldots 10$ (Strongly agree) & 10 & 4 & 8.23 & 9.00 & 1.99 \\
\hline Q7 & $\begin{array}{l}\text { In the first phase that required you to plan a traverse route, the } \\
\text { interfaces used for placing station markers and receiving in- } \\
\text { structions/feedbacks from the Mission Control (NASA officer) } \\
\text { were well designed to guide you through the steps of the route } \\
\text { planning. }\end{array}$ & (Strongly disagree) $1 \ldots 10$ (Strongly agree) & 10 & 5 & 8.47 & 9.00 & 1.48 \\
\hline Q8 & $\begin{array}{l}\text { In the second phase that required you to install devices on the } \\
\text { vehicle, the way of playing motivated you to learn the function- } \\
\text { ality of individual devices. }\end{array}$ & (Strongly disagree) $1 \ldots 10$ (Strongly agree) & 10 & 3 & 7.58 & 8.50 & 2.46 \\
\hline Q9 & $\begin{array}{l}\text { In the third phase that required you to drive the vehicle on the } \\
\text { lunar surface, how long did it take you to understand the me- } \\
\text { chanical differences between driving the lunar rover and driving } \\
\text { a regular car? (Note: do not consider how well you drove it; just } \\
\text { consider how much time it took you to understand the driving } \\
\text { method.) }\end{array}$ & (Immediately) $1 \ldots 10$ (Takes forever) & 8 & 1 & 4.03 & 3.50 & 2.46 \\
\hline Q10 & $\begin{array}{l}\text { During the play, did you feel panic or depressed at any moment? } \\
\text { If "Yes", at what playing stage you felt? }\end{array}$ & - & - & - & - & - & - \\
\hline
\end{tabular}

Some participants had a mild difficulty to follow text-based instructions.

Educational values. The educational value of the game is recognized by the participants. One participant commented that the game might be also suitable for young audience, as she said "It was pretty fun and very educational. I really enjoyed it and if the target audience for the game is elementary and middle school children, I'm sure it will be quite enjoyable."

As regards Q3 and Q4, participants provided mixed feedback: some participants were willing to learn more about the lunar exploration missions; some participants were more interested in mechanics of the LRV; and some participants felt that they experienced an event that they otherwise would not know, and they would like to know more about what other things the astronauts did on the Moon. One participant said that "I would like to know how long an astronaut drove the car per day and what the most challenging task was on the Moon," which are not presented in the current version of the game. One participant commented that "I did feel like doing more research on how the rover technology has improved since then and the new improvements that they have made." Another participant expressed that he gained interest in mechanical engineering. Some participants seemed to become more interested in geomorphology, as one said that "I want to know more about the ground on the Moon which may make driving there easier. I feel curious about the environment on the Moon and on the Mars."

The events in the original lunar exploration missions involve the contributions from experts in science, technology, engineering, physics, and mathematics. Our game represents such in- terdisciplinary concepts. While participants were learning the events and getting to know more space science, the game also successfully made them interested in various areas in related disciplines. The participants' answers to Q3 and Q4 indicate the game makes itself useful in delivering a positive impact on general STEM education.

Positive comments in response to Q5 and Q6 highlight the game design. One participant commented that "There weren't many distractions on the screen and that helped to make the game easier." But some participants felt that there were too many texts on the interface requiring intensive reading. While the participants agreed that text-based instructions were effective for learning how to play, they would prefer a graphical tutorial to guide them through the process. Positive comments also highlight other gameplay elements, as one participant said that "The movement of rover, the movement of camera, the particle effects in milestones are quite pleasing." Using the flight stick added difficulties to the play since it is not a common controller, but many participants commented that it was the best part of the game. Some participants reported that they did not know how to use it at the beginning, but it was easy to learn and consistent to the natural way of rover driving. According to the participants' answers to Q5 and Q6, The unique interaction provided by the flight stick well represented the steering system of the LRV at driving.

Evaluation of individual gameplay phases. As regards Q7 for the phase of planning a traverse route, we received positive comments. Many participants said the interface promoted learning and was fairly easy to use. One participant commented 
that "I have a much greater understanding of how rover trips were planned." As regards Q8, the Equipment Loading phase was very informational. Interactions with the 3D LRV and individual devices motivated the participants to further observe the scientistic usages. Reading device descriptions required patience. As regards Q9 about driving the LRV, the participants gained the knowledge that "there is a big difference between driving the lunar rover and driving a car." By playing this game, they quickly learned the technical differences between the lunar rover and a regular car, which would not be understood so fast by reading materials. One participant commented that "the overheating was frustrating, but I knew to drive slowly otherwise I would not know without that." The game helped them understand high temperatures on the motors and batteries are critical factors for driving.

Depressive and panic feelings at driving were reported. Some participants felt anxious and tense when the LRV was out of control (e.g., fell off the crater or upside down). This experience was consistent to the fact that the astronauts would have in the Apollo missions. The participants were able to gain the knowledge and understand the challenges the astronauts had after they experienced the same in the game.

\section{Conclusion and Future Work}

The paper presented the gamification of Apollo lunar exploration missions and the evaluation of using the game to promote STEM learning. We described the design and development of the game based on the missions the astronauts executed in 1960s and 1970s. The technical solutions for generating the high quality lunar terrain and real-time rendering complex geometric models were also addressed. The usability study was done by a multidisciplinary group of participants, who suggested a number of positive comments and improvements for future development.

In the future, we will gamify more Apollo missions. We are currently working on the gamification of Apollo 17 mission. We would like users to learn about the evolution of the sequence of Apollo missions, which would be important to the history of space technology. We would like to improve the game according to the comments we received. We plan to add cut scenes or video clips to show the actions of astronauts collecting soil samples or setting up science experiments. More rock obstacles will be added to make the driving more challenging. We plan to modify the game for elementary and middle school students by creating child-friendly controls and interfaces (e.g., replacing text-based instructions with graphical tutorials). We also plan to perform a quantitative assessment to identify the relationship among the number of times of play, the improvement of play score, and learning efficiency.

\section{Acknowledgement}

This work was supported in part by NSF under grant number CNS-1464323 and in part by UAH CCFR program under grant number 254342 . We gratefully acknowledge the support of NVIDIA Corporation with the donation of a GPU card used in this project. We thank anonymous reviewers for their comments. We thank the participants for their participations in the usability study. We thank Ron Creel, member of the Apollo Lunar Roving Vehicle Team, for his requirements for the game design. We thank Don McMillan for providing the Lunar Rover 3D surface model for use in the game. We acknowledge the collaborative efforts between UAH and LUROVA ${ }^{\mathrm{TM}}$. We thank Lloyd Hillman and Michael Vance for their contributions to the early prototype of this game. We thank Samyam Acharya for his contribution to the game development and his help on the usability study. We thank Vinny Argentina for his valuable feedback.

[1] E. Poliakoff, T. L. Webb, What factors predict scientists' intentions to participate in public engagement of science activities?, Science Communication 29 (2) (2007) 242-263. doi:10.1177/1075547007308009.

[2] G. Hagger-Johnson, P. Hegarty, M. Barker, C. Richards, Public engagement, knowledge transfer, and impact validity, Journal of Social Issues 69 (4) (2013) 664-683. doi:10.1111/josi.12035.

URL http://dx.doi.org/10.1111/josi.12035

[3] M. Hilton, M. A. Honey, et al., Learning science through computer games and simulations, National Academies Press, 2011.

[4] E. Afari, J. M. Aldridge, B. J. Fraser, M. S. Khine, Students' perceptions of the learning environment and attitudes in game-based mathematics classrooms, Learning Environments Research 16 (1) (2012) 131-150. doi:10.1007/s10984-012-9122-6.

[5] H.-Y. Sung, G.-J. Hwang, A collaborative game-based learning approach to improving students' learning performance in science courses, Computers \& Education 63 (2013) 43 - 51. doi:http://dx.doi.org/10.1016/j.compedu.2012.11.019.

[6] S. Deterding, D. Dixon, R. Khaled, L. Nacke, From game design elements to gamefulness: Defining "gamification", in: Proceedings of the 15th International Academic MindTrek Conference: Envisioning Future Media Environments, MindTrek '11, ACM, New York, NY, USA, 2011, pp. 9-15. doi:10.1145/2181037.2181040.

URL http: //doi .acm.org/10.1145/2181037.2181040

[7] S. Deterding, M. Sicart, L. Nacke, K. O’Hara, D. Dixon, Gamification. using game-design elements in non-gaming contexts, in: $\mathrm{CHI}$ '11 Extended Abstracts on Human Factors in Computing Systems, CHI EA '11, ACM, New York, NY, USA, 2011, pp. 2425-2428. doi:10.1145/1979742.1979575.

URL http://doi .acm.org/10.1145/1979742.1979575

[8] C. Murphy, Why games work and the science of learning, in: Interservice, Interagency Training, Simulations, and Education Conference, Citeseer, 2011, pp. 260-272.

[9] N. S. Foundation. National center for science and engineering statistics [online] (2016).

[10] S. J. Markovich. Space exploration and u.s. competitiveness [online] (December 2014) [cited 02/06/2016].

[11] M. Selvakumar, M. Storksdieck, Portal to the public: Museum educators collaborating with scientists to engage museum visitors with current science, Curator: The Museum Journal 56 (1) (2013) 69-78. doi:10.1111/cura.12007.

URL http://dx.doi .org/10.1111/cura.12007

[12] E. Dawson, Equity in informal science education: developing an access and equity framework for science museums and science centres, Studies in Science Education 50 (2) (2014) 209247. arXiv:http://dx.doi.org/10.1080/03057267.2014.957558, doi:10.1080/03057267.2014.957558.

URL http://dx.doi.org/10.1080/03057267.2014.957558

[13] Y. Yang, J. Bao, Y. Jin, Y. Cheng, A virtual simulation environment for lunar rover: Framework and key technologies, International Journal of Advanced Robotic Systems 5 (2) (2008) 201-208.

[14] H. Gao, Z. Deng, L. Ding, M. Wang, Virtual simulation system with path-following control for lunar rovers moving on rough terrain, Chinese Journal of Mechanical Engineering 25 (1) (2012) 38-46. doi:10.3901/CJME.2012.01.038.

URL http://dx.doi.org/10.3901/CJME. 2012.01.038 
[15] D. Djaouti, J. Alvarez, J.-P. Jessel, O. Rampnoux, Serious Games and Edutainment Applications, Springer London, London, 2011, Ch. Origins of Serious Games, pp. 25-43. doi:10.1007/978-1-4471-2161-9_3.

[16] B. Sawyer, D. Rejeski, Serious games: Improving public policy through game-based learning and simulation, Woodrow Wilson International Center for Scholars (2002).

[17] S. Ghamari-Tabrizi, Simulating the unthinkable: Gaming future war in the 1950s and 1960s, Social Studies of Science 30 (2) (2000) 163-223. arXiv:http://sss.sagepub.com/content/30/2/163.full.pdf+html, doi:10.1177/030631200030002001.

URL http://sss. sagepub.com/content/30/2/163. abstract

[18] K. Squire, Changing the game: What happens when video games enter the classroom, Innovate: Journal of online education 1 (6).

[19] W. R. Watson, C. J. Mong, C. A. Harris, A case study of the in-class use of a video game for teaching high school history, Computers \& Education 56 (2) (2011) 466-474.

[20] F. Ricciardi, L. T. D. Paolis, A comprehensive review of serious games in health professions, Int. J. Comput. Games Technol. 2014 (2014) 9:9-9:9. doi:10.1155/2014/787968.

[21] S. Luz, M. Masoodian, R. R. Cesario, M. Cesario, Using a serious game to promote community-based awareness and prevention of neglected tropical diseases, Entertainment Computing (2015) doi:http://dx.doi.org/10.1016/j.entcom.2015.11.001.

[22] J. Froschauer, M. Arends, D. Goldfarb, D. Merkl, Towards an online multiplayer serious game providing a joyful experience in learning art history, in: Games and Virtual Worlds for Serious Applications (VSGAMES), 2011 Third International Conference on, 2011, pp. 160-163. doi:10.1109/VS-GAMES.2011.47.

[23] M. Romero, M. Usart, E. Almirall, Serious games in a finance course promoting the knowledge group awareness, in: EDULEARN11 Proceedings, 3rd International Conference on Education and New Learning Technologies, IATED, 2011, pp. 3490-3492.

[24] R. Hromek, S. Roffey, Promoting social and emotional learning with games: "it's fun and we learn things", Simulation \& Gamingdoi: $10.1177 / 1046878109333793$.

[25] S. Arnab, K. Brown, S. Clarke, I. Dunwell, T. Lim, N. Suttie, S. Louchart, M. Hendrix, S. de Freitas, The development approach of a pedagogicallydriven serious game to support relationship and sex education (rse) within a classroom setting, Computers \& Education 69 (2013) 15 - 30 . doi:http://dx.doi.org/10.1016/j.compedu.2013.06.013.

[26] J. Froschauer, I. Seidel, M. Gärtner, H. Berger, D. Merkl, Design and evaluation of a serious game for immersive cultural training, in: Virtual Systems and Multimedia (VSMM), 2010 16th International Conference on, 2010, pp. 253-260. doi:10.1109/VSMM.2010.5665978.

[27] E. M. Raybourn, Applying simulation experience design methods to creating serious game-based adaptive training systems, Interacting with Computers 19 (2) (2007) 206-214. arXiv:= doi:10.1016/j.intcom.2006.08.001

[28] P. Backlund, H. Engstrom, C. Hammar, M. Johannesson, M. Lebram, Sidh - a game based firefighter training simulation, in: Information Visualization, 2007. IV '07. 11th International Conference, 2007, pp. 899907. doi:10.1109/IV.2007.100.

[29] T. Hodgson, J. Eisenlohr, et al., Nasa-learning technologies-research.

[30] M. J. Mayo, Video games: A route to large-scale stem education?, Science 323 (5910) (2009) 79-82. arXiv:http://science.sciencemag.org/content/323/5910/79.full.pdf, doi:10.1126/science. 1166900

URL http://science.sciencemag. org/content/323/5910/79

[31] L. M. Miller, C.-I. Chang, S. Wang, M. E. Beier, Y. Klisch, Learning and motivational impacts of a multimedia science game, Computers \& Education 57 (1) (2011) 1425 - 1433. doi:http://dx.doi.org/10.1016/j.compedu.2011.01.016.

URL http://www.sciencedirect.com/science/article/pii/S03601

[32] L. Miller, Role-play games as a tool for stem career inspiration and the development of scientific possible selves, in: Proceedings of Global Learn 2015, Association for the Advancement of Computing in Education (AACE), Berlin, Germany, 2015, pp. 385-389.

URL https : //www . learntechlib.org/p/150883

[33] A. Yusoff, R. Crowder, L. Gilbert, G. Wills, A conceptual framework for serious games, in: Advanced Learning Technologies, 2009. ICALT 2009. Ninth IEEE International Conference on, 2009, pp. 21-23.
doi:10.1109/ICALT.2009.19.

[34] W. Li, T. Grossman, G. Fitzmaurice, Gamicad: A gamified tutorial system for first time autocad users, in: Proceedings of the 25th Annual ACM Symposium on User Interface Software and Technology, UIST '12, ACM, New York, NY, USA, 2012, pp. 103-112. doi:10.1145/2380116.2380131. URL http://doi .acm.org/10.1145/2380116.2380131

[35] B. Kim, Designing gamification in the right way, Library Technology Reports 51 (2) (2015) 29-35.

[36] K. Dergousoff, R. L. Mandryk, Mobile gamification for crowdsourcing data collection: Leveraging the freemium model, in: Proceedings of the 33rd Annual ACM Conference on Human Factors in Computing Systems, CHI '15, ACM, New York, NY, USA, 2015, pp. 1065-1074. doi:10.1145/2702123.2702296. URL http: //doi .acm.org/10.1145/2702123.2702296

[37] W. Muehlberger, Apollo 16 traverse planning and field procedures, Geological Survey Professional Paper 1048 (1981) 10-20.

[38] A. Young, Lunar and planetary rovers: the wheels of Apollo and the quest for Mars, Springer Science \& Business Media, 2007.

[39] L. Boeing, Systems engineering, lunar rover operations handbook, doc, Tech. rep., LS006-002-2H, Huntsville, Alabama, 19 April (1971).

[40] P. B. Hager, Dynamic thermal modeling for moving objects on the moon, Dissertation, Technische Universität München, München (2013).

[41] R. Creel, Lurova - from render engine to thermal model, in: Thermal and Fluids Analysis Workshop, Glenn Reseearch Center, Cleveland, $\mathrm{OH}$, 2014.

[42] L. Tenenbaum, R. Jackson, M. Greene, Techniques for integrating the animations, multimedia, and interactive features of nasa's climate change website, climate change: Nasa's eyes on the earth, into the classroom to advance climate literacy and encourage interest in stem disciplines, in: AGU Fall Meeting Abstracts, Vol. 1, 2009, p. 0541.

[43] G. Chin, S. Brylow, M. Foote, J. Garvin, J. Kasper, J. Keller, M. Litvak, I. Mitrofanov, D. Paige, K. Raney, et al., Lunar reconnaissance orbiter overview: The instrument suite and mission, Space Science Reviews 129 (4) (2007) 391-419.

[44] NASA, Lunar mapping and modeling portal [cited 02/20/2016]. URL http: //pub. lmmp.nasa.gov/LMMPUI/LMMP_CLIENT/LMMP.htm]

[45] K. N. Burns, E. Speyerer, M. Robinson, T. Tran, M. Rosiek, B. Archinal, E. Howington-Kraus, et al., Digital elevation models and derived products from lroc nac stereo observations, ISPRS-International Archives of the Photogrammetry, Remote Sensing and Spatial Information Sciences 1 (2012) 483-488

[46] F. Cagnacci, F. Urbano, Managing wildlife: a spatial information system for gps collars data, Environmental Modelling \& Software 23 (7) (2008) 957-959.

[47] X. Sun, P. Rosin, R. Martin, F. Langbein, Fast and effective featurepreserving mesh denoising, Visualization and Computer Graphics, IEEE Transactions on 13 (5) (2007) 925-938. doi:10.1109/TVCG.2007.1065.

[48] D. McMillan, Virtual lunar rover vehicle (2005) [cited 02/22/2016] URL https : //www.hq.nasa.gov/alsj/VirtualLRV.html

[49] H. Hoppe, Progressive meshes, in: Proceedings of the 23rd Annual Conference on Computer Graphics and Interactive Techniques, SIGGRAPH '96, ACM, New York, NY, USA, 1996, pp. 99-108. doi:10.1145/237170.237216.

URL http://doi .acm.org/10.1145/237170.237216

[50] C. Peng, Y. Cao, A gpu-based approach for massive model rendering with frame-to-frame coherence, Computer Graphics Forum 31 (2pt2) (2012) 393-402. doi:10.1111/j.1467-8659.2012.03018.x. URL http: //dx.doi.org/10.1111/j.1467-8659.2012.03018.x

[51] C. Peng, Y. Cao, Parallel lod for cad model rendering with effective gpu memory usage, Computer-Aided Design and Applications 13 (2) (2016) 173-183. arXiv:http://dx.doi.org/10.1080/16864360.2015.1084184, doi:10.1080/16864360.2015.1084184

315110D339tp: //dx . doi .org/10.1080/16864360.2015.1084184

[52] G. D. Ruxton, The unequal variance t-test is an underused alternative to student's t-test and the mannwhitney $\mathrm{u}$ test, Behavioral Ecology 17 (4) (2006) 688-690. arXiv:http://beheco.oxfordjournals.org/content/17/4/688.full.pdf+html, doi:10.1093/beheco/ark016.

URL http://beheco.oxfordjournals .org/content/17/4/688. short 